\title{
H $\alpha$ KINEMATICS OF THE ISOLATED INTERACTING GALAXY PAIR KPG 486 (NGC 6090)
}

\author{
M. M. Sardaneta ${ }^{1}$, M. Rosado ${ }^{2}$, and M. Sánchez-Cruces ${ }^{1}$ \\ Received March 19 2019; accepted December 172019
}

\begin{abstract}
In optical images, the barely studied isolated interacting galaxy pair KPG 486 (NGC 6090) displays similar features to the Antennae (NGC 4038/39) galaxy pair. To compare the distribution of ionized hydrogen gas, morphology and kinematic and dynamic behaviour of both galaxy pairs, we present observations in the $\mathrm{H} \alpha$ emission line of NGC 6090 acquired with the scanning Fabry-Perot interferometer, PUMA. For each galaxy in NGC 6090 we obtained several kinematic parameters, its velocity field and its rotation curve. We also analysed some of the perturbations induced by their encounter. We verified the consistency of our results by comparing them with kinematic results from the literature. The comparison of our results on NGC 6090 with those obtained in a previous similar kinematic analysis of the Antennae highlighted great differences between these galaxy pairs.
\end{abstract}

\section{RESUMEN}

En imágenes del óptico, el par de galaxias aislado poco estudiado, KPG 486 (NGC 6090), muestra características similares al par de galaxias Las Antenas (NGC 4038/39). Para comparar la distribución del gas ionizado, morfología y comportamiento cinemático y dinámico entre ambos pares de galaxias, se presentan observaciones en la línea de emisión H $\alpha$ de NGC 6090 adquiridas con el interferómetro Fabry-Perot de barrido, PUMA. Para cada galaxia en NGC6090 se obtuvieron varios parámetros cinemáticos, su campo de velocidades y su curva de rotación. Además, se analizaron algunas de las perturbaciones inducidas por su encuentro. Se verificó la consistencia de estos resultados comparándolos con los de la literatura. La comparación de los resultados de NGC 6090 con los de un análisis cinemático similar previo para las Antenas destacó grandes diferencias entre estos pares de galaxias.

Key Words: galaxies: individual: NGC 6090 - galaxies: interactions — galaxies: kinematics and dynamics — methods: data analysis — techniques: interferometric

\section{INTRODUCTION}

Instead of evolving in isolation, galaxies are found in clusters and groups, and they can interact quite strongly with their nearby companions. These interactions can have a profound impact on the properties of galaxies, resulting in intense bursts of star formation, the onset of quasar-like activity in galactic nuclei and perhaps even the complete transformation of spiral galaxies into elliptical galaxies. Studies of galaxies in the early universe show a significant fraction of interacting and merging systems, and theo-

\footnotetext{
${ }^{1}$ Aix Marseille Univ, CNRS, CNES, LAM, Marseille, France.

${ }^{2}$ Instituto de Astronomía, UNAM, Ciudad de México, México.
}

ries of cosmological structure formation indicate that most galaxies have had some form of strong interaction during their lifetime. Rather than being rare events, galaxy interactions may be the dominant process shaping the evolution of the galaxy population in general (Mihos 2000).

The evolution and interaction of galaxies is governed by gravitational effects. Morphologically, in the interacting galaxies there are large bridges and tails, stellar bars and/or increased spiral structures and, commonly, the bodies of galaxies are distorted (Schweizer 1986). Toomre \& Toomre (1972), through numerical simulations, established that the gravitational interaction with another galaxy may be 
the source not only of spiral structures, but also of the filamentary structure, which they called 'tidal tails'; thus, gravity is solely responsible for these large and thin tails and several other deformations seen in several surveys (e.g. Arp 1966; Karachentsev 1987; Vorontsov-Velyaminov et al. 2001).

Numerical simulations show that during a merger, tidal forces from a companion galaxy trigger the formation of a bar in the disc of a perturbed galaxy (Noguchi 1987; Salo \& Laurikainen 2000a,b; Renaud et al. 2015). The bar acts to trigger starburst activity by rapidly funnelling large amounts of gas to the nuclear region (Barnes \& Hernquist 1992; Dinshaw et al. 1999; Gabbasov et al. 2014; Martin \& Soto 2016). When a bar is clearly observable in the stellar component, the gas dynamics shows very distinctive characteristics, corresponding to elliptical and non-circular orbits; the isovelocities contour curves are deformed symmetrically. Hence, the inclination of the central isovelocity contour curve along the minor axis is one of the main features used to identify a bar (Bosma 1981; Combes et al. 2002).

The simplest case in the process of galaxy interactions is that of isolated galaxy pairs, which are systems composed of two galaxies located so close in space that the gravitational effect of their nearest neighbours can be neglected relative to the gravitational effects exerted between each other (FuentesCarrera et al. 2004; Rosado et al. 2011; Gabbasov et al. 2014). Systematic research on double galaxies provides us with important information about the conditions of formation and properties of the evolution of galaxies (Karachentsev 1987).

Obtaining kinematic information from interacting galaxies is useful for understanding the effect that the interaction can have on each of the members of the pair (Fuentes-Carrera et al. 2004, 2007; Repetto et al. 2010). In highly disturbed velocity fields, double nuclei, double kinematic gas components, and high amplitude discrepancies between both sides of the rotation curves imply strong galaxygalaxy interactions or mergers. On the other hand, stellar and gaseous major axes misalignments and tidal tails suggest collisions that may not always lead to merging (Amram et al. 2003; Torres-Flores et al. 2014).

From the observational point of view, most of the kinematic work on interacting galaxies has been carried out using long-slit spectroscopy along certain positions (e.g. Günthardt et al. 2016), restricting kinematic information to only a few points on the galaxy. However, for an asymmetric perturbed system, it is important to obtain the kinematic in- formation from large portions of the disc, using observational techniques such as integral field spectroscopy with a scanning Fabry-Perot interferometer (FP). In this way, the extended kinematic information can help us determine the interaction process which has been produced on each of the members of the interacting system, in addition to the fact that, sometimes the axial symmetry of each galaxy is lost during the interaction (Fuentes-Carrera et al. 2004, 2015; Epinat et al. 2008).

\subsection{NGC 6090}

The isolated interacting galaxy pair KPG 486 (NGC 6090) has been described as a double nuclei system with an asymmetric disc and two long tidal tails of $\approx 60 \mathrm{kpc}$ in length (e.g. Dinshaw et al. 1999). At optical wavelengths, this galaxy system looks like the NGC 4038/39 galaxy system (The Antennae) (e.g. Toomre \& Toomre 1972; Hummel et al. 1987; Martin et al. 1991; Mazzarella \& Boroson 1993; Dinshaw et al. 1999; Bryant \& Scoville 1999). This feature can be observed in the upper panel of Figure 1 which shows the optical image of NGC 6090 taken from the Digitized Sky Survey (DSS) in a field of view of 4 arcmin.

In radio-wavelengths NGC 6090 appears as a pair of interacting spirals separated by 0.14 arcsec, with nuclei in contact and with enormous curved wings (Martin et al. 1991). Meanwhile, molecular gas appears elongated and aligned along the direction of the nuclei as a rotating disc (Wang et al. 2004) or ring (Bryant \& Scoville 1999; Sugai et al. 2000). The position angle of that disc is $\approx 60^{\circ}$ with a major axis length of $\approx 3.4$ arcsec; the $\mathrm{CO}$ source appears to peak between the radio nuclei rather than on one of them (Bryant \& Scoville 1999) and the molecular gas component does not appear to belong to any of the galaxies in the system based on kinematics (Wang et al. 2004).

Due to the interaction evidence that NGC 6090 shows it was defined as a merger by Chisholm et al. (2015). But NGC 6090 has also been defined as a galaxy system in an intermediate stage of merging or pre-merging because, in addition of its two nuclei and tidal tails, the galaxy system has an identifiable bridge (Miralles-Caballero et al. 2011). In the near-infrared, the galaxies that make up NGC 6090 are seen as follows: NGC $6090 \mathrm{NE}$ has a distorted spiral structure and evidence of a stellar bar, and NGC 6090 SW looks like an irregular galaxy; the position of its nucleus is still under discussion (Dinshaw et al. 1999; Cortijo-Ferrero et al. 2017). In Table 1 we list the general parameters of NGC 6090 which 


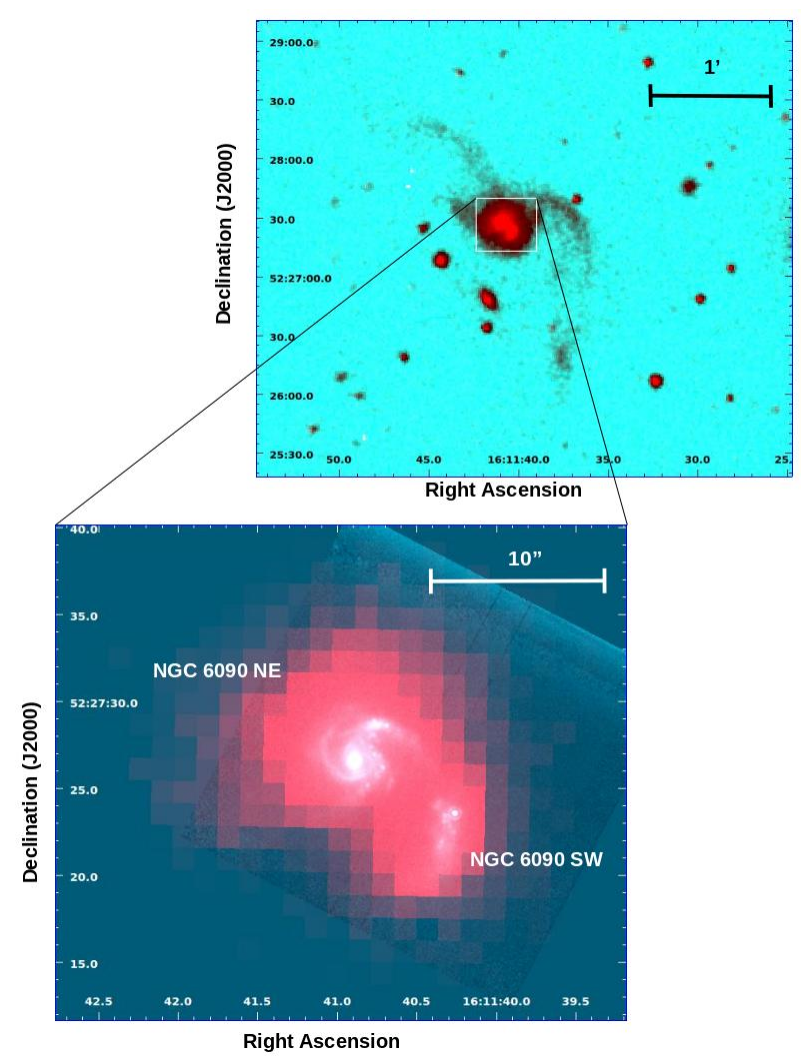

Fig. 1. Top: Image from the Digitized Sky Survey (DSS) in a 4 arcmin field of view centred on the galaxy system NGC 6090 over which, superposed in red, is shown the $\mathrm{H} \alpha$ direct image, taken with a $6819 \AA$ filter with a FWHM of $86 \AA$ (obtained from the PUMA in its direct image mode at the $2.1 \mathrm{~m}$ telescope from the Observatorio Astronómico Nacional in San Pedro Mártir, OANSPM, Baja California, México). Bottom: $2.22 \mu \mathrm{m}$ image from the Near-Infrared Camera Multi-Object on the Hubble Space Telescope (NICMOS/HST) over which, superposed in red, is shown the $\mathrm{H} \alpha$ direct image from OANSPM in a 28 arcsec field of view centred on NGC 6090. The color figure can be viewed online.

hitherto have been reported only for a single galactic system.

To investigate the distribution of ionized hydrogen gas, the morphology, and kinematic and dynamic behaviour of the galaxy system NGC 6090, we obtained a FP data cube spectrally centreded on the $\mathrm{H} \alpha$ emission line of the system. In this paper we present the analysis of the $\mathrm{H} \alpha$ image, the velocity field and dispersion velocity map of the pair of galaxies NGC 6090, as well as the rotation curves obtained for each member. Lastly, we comment on our results and those found in the literature, and compare
TABLE 1

\section{PARAMETERS OF THE GALAXY SYSTEM NGC 6090}

\begin{tabular}{|c|c|}
\hline Parameters & NGC 6090 system \\
\hline \multirow[t]{2}{*}{ Coordinates (J2000) } & $\alpha=16^{\mathrm{h}} 11^{\mathrm{m}} 40.7^{\mathrm{s}}$ \\
\hline & $\delta=+52^{\circ} 27^{\prime} 24^{\prime \prime} . \mathrm{a}$ \\
\hline \multirow[t]{4}{*}{ Other names } & $\mathrm{KPG} 486^{\mathrm{a}, \mathrm{c}}$ \\
\hline & NGC $6090^{a}$ \\
\hline & UGC $10267^{\mathrm{a}}$ \\
\hline & $\operatorname{Mrk} 496^{\mathrm{a}}$ \\
\hline \multirow[t]{4}{*}{ Morphological type } & G Pair ${ }^{a}$ \\
\hline & Multiple galaxy ${ }^{\mathrm{b}}$ \\
\hline & Merger, double nucleus ${ }^{\mathrm{e}}$ \\
\hline & Merger ${ }^{g}$ \\
\hline Mean heliocentric & $8906^{a}$ \\
\hline Radial velocity $\left(\mathrm{km} \mathrm{s}^{-1}\right)$ & $8855^{\mathrm{b}}$ \\
\hline \multirow[t]{4}{*}{ Distance (Mpc) } & $122^{\mathrm{d}, *}$ \\
\hline & $123.3^{\mathrm{e}, *}$ \\
\hline & $128^{\mathrm{g}, * *}$ \\
\hline & $127.7 \mathrm{~h}, * *$ \\
\hline $\mathrm{L}_{\mathrm{IR}}\left(L_{\odot}\right)$ & $3 \times 10^{11 \mathrm{f}}$ \\
\hline $\mathrm{m}_{b}$ & $14.36^{\mathrm{a}}$ \\
\hline $\mathrm{D}_{25 / 2}(\operatorname{arcmin})$ & $4.36^{\mathrm{b}}$ \\
\hline \multicolumn{2}{|l|}{ Photometric nuclear } \\
\hline Separation (arcsec) & $5.4^{\mathrm{e}}$ \\
\hline
\end{tabular}

${ }^{a}$ NASA/IPAC (2014) (NASA/IPAC Extragalactic Data Base (NED) is operated by the Jet Propulsion Laboratory, California Institute of Technology, under contract with the National Aeronautics and Space Administration. https://ned.ipac.caltech.edu/).

${ }^{\mathrm{b}}$ HyperLeda (HyperLeda: http://leda.univ-lyon1.fr/).

${ }^{c}$ Karachentsev (1987).

${ }^{\mathrm{d}}$ Condon et al. (1990).

${ }^{\mathrm{e}}$ Bryant \& Scoville (1999).

${ }^{\mathrm{f}}$ Acosta-Pulido et al. (1996).

${ }^{\mathrm{g}}$ Chisholm et al. (2015).

${ }^{\mathrm{h}}$ Cortijo-Ferrero et al. (2017).

${ }^{*}$ Distance $D=v / H_{0}$ computed using $H_{0}=$ $75 \mathrm{~km} \mathrm{~s}^{-1} \mathrm{Mpc}^{-1}$.

${ }^{* *}$ Distance obtained from $z=0.02930$ with $H_{0}=$ $70 \mathrm{~km} \mathrm{~s}^{-1} \mathrm{Mpc}^{-1}$

the kinematic characteristics of the galaxy system NGC 6090 with those of the Antennae made by Amram et al. (1992).

This paper is organized as follows: in $\S 2$ there is an overview of the observational parameters and the reduction process; in $\S 3$ we present the morphological features of NGC 6090 observed from its $\mathrm{H} \alpha$ monochromatic and continuum maps; $\S 4$ is devoted 
TABLE 2

INSTRUMENTAL AND OBSERVATIONAL PARAMETERS

\begin{tabular}{lc}
\hline Parameter & Value \\
\hline Telescope & $2.1 \mathrm{~m}(\mathrm{OAN}, \mathrm{SPM})$ \\
Instrument & PUMA \\
Detector & Site3 CCD \\
Detector size & $2048 \times 2048 \mathrm{pix}$ \\
Image scale (binning $4 \times 4)$ & $1.27 \mathrm{arcsec} / \mathrm{pix}$ \\
Scanning FP interferometer & $\mathrm{ET}-50$ \\
Finesse & $\approx 10$ \\
FP interference order at H $\alpha$ & 330 \\
Free spectral range at H $\alpha$ & $19.9 \AA\left(909 \mathrm{~km} \mathrm{~s}{ }^{-1}\right)$ \\
Spectral sampling & \\
$\quad$ resolution at H $\alpha$ & $0.97 \AA\left(44.1 \mathrm{~km} \mathrm{~s}{ }^{-1}\right)$ \\
Interference filter & $8819 \AA(\mathrm{FWHM}=86 \AA)$ \\
Total exposure time & $72 \mathrm{~min}$ \\
Calibration line & $6717 \AA(\mathrm{Ne})$ \\
Average seeing & $1.67 \mathrm{arcsec}$ \\
\hline
\end{tabular}

to the kinematic analysis made from our FP data. We show the derived velocity fields, the associated rotation curve of each member of the galaxy system NGC 6090 and we analyse the non-circular motions of each galaxy through the velocity dispersion map of the system; $\S 5$ is dedicated to the dynamical analysis, including the computation of the mass of each galaxy. A discussion is presented in $\S 6$, and our conclusions are given in $\S 7$.

In this paper, we considered $H_{0}=75 \mathrm{~km} \mathrm{~s}^{-1} \mathrm{Mpc}^{-1} \quad$ (Condon et al. 1990) and adopted a distance of $118 \mathrm{Mpc}$ for NGC 6090 .

\section{OBSERVATIONS AND DATA REDUCTIONS}

Our observations of NGC 6090 were performed in July 2015 with the $f / 7.5$ Cassegrain focus at the $2.1 \mathrm{~m}$ telescope at the Observatorio Astronómico Nacional in San Pedro Mártir, Baja California, México (OAN-SPM) using the scanning Fabry-Perot interferometer, PUMA (Rosado et al. 1995). PUMA is a focal reducer built at the Instituto de AstronomíaUNAM and used to obtain images and FabryPerot interferometry of extended emission cosmical sources. The FP used is an ET-50 (Queensgate Instruments) with a servostabilization system; it has a free spectral range of $19.9 \AA\left(909 \mathrm{~km} \mathrm{~s}^{-1}\right)$ at $\mathrm{H} \alpha$. The effective finesse of PUMA is $\approx 24$ which implies a sampling spectral resolution in $\mathrm{H} \alpha$ of $0.414 \AA$ $\left(19.0 \mathrm{~km} \mathrm{~s}^{-1}\right)$ achieved by scanning the interferometer free spectral range through 48 different equally spaced channels (Rosado et al. 1995). However, due to the parallelism of the FP mirrors during the data acquisition for the observations, particularly of NGC 6090, the measured finesse for this data cube was $\approx 10$ which leads to a sampling spectral resolution of $0.97 \AA\left(44.1 \mathrm{~km} \mathrm{~s}^{-1}\right)$ at $\mathrm{H} \alpha$.

We used a $2048 \times 2048$ CCD detector with a pixel scale of $0.317 \operatorname{arcsec}$ and we set a $4 \times 4$ binning to enhance the signal, obtaining a $512 \times 512$ pixel window inside of a field of view of $10 \mathrm{arcmin}$. So, we obtained a final spatial sampling per pixel of 1.27 arcsec which is slightly better than the average seeing of 1.67 arcsec. To isolate the redshifted $\mathrm{H} \alpha$ emission line an interference filter centred at $6819 \AA$ with FWHM of $86 \AA$ was used. Thus, we obtained an $\mathrm{H} \alpha$ data cube with a total exposure time of $72 \mathrm{~min}-$ utes (90 s per channel).

In order to avoid phase wavelength dependence of the FP layers it is necessary to calibrate the data cube in wavelength. So, we used a Ne lamp whose line at $6717 \AA$ is close to the redshifted nebular wavelength to obtain a calibration cube at the end of the observation. The parabolic phase map was computed from the calibration cube in order to obtain the reference wavelength for the line profile observed inside each pixel. The instrumental and observational parameters are listed in Table 2.

For the data reduction and analysis we used the $\mathrm{ADHOCw}^{3}$ software to make the standard corrections to the cube: removal of cosmic rays and bias subtraction, subtraction of the $\mathrm{OH}$ sky lines at 6828.5 $\AA, 6842.2 \AA$ and $6863.9 \AA$ (Chamberlain 1961; Krassovsky et al. 1962) and application of a spectral Gaussian smoothing with $\sigma=57 \mathrm{~km} \mathrm{~s}^{-1}$ in the data cube. We applied IRAF ${ }^{4}$ tasks such as IMEXAMINE to determine the average seeing of our data and MSCSETWCS to add the World Coordinate System (WCS) to the resulting maps of NGC 6090. To complete the data analysis we used our own Python scripts.

The FP scanning process allows us to obtain a flux value in arbitrary units at pixel level for each of the 48 scanning steps. The calibration in wavelength was fixed for each profile at each pixel using the calibration data cube. Thus, the intensity profile found throughout the scanning process contains information about the monochromatic emission (ionized gas emitting at $\mathrm{H} \alpha$ ), the continuum emission of the object, as well as the velocity of the ionized gas.

\footnotetext{
${ }^{3}$ http://cesam.lam.fr/fabryperot/index/softwares developed by J. Boulesteix.

"IRAF: "Image Reduction and Analysis Facility" http:// iraf.noao.edu/.
} 
The computation of the image of the continuum was made considering the average of the 3 lowest intensities of the 48 channels of the cube (e.g. Vollmer et al. 2000; Fuentes-Carrera et al. 2004; Repetto et al. 2010; Rosado et al. 2013; Cárdenas-Martínez \& Fuentes-Carrera 2018). For the monochromatic image, the intensity of the $\mathrm{H} \alpha$ line was obtained by integrating the maximum value of the line profile for each pixel.

ADHOCw calculates the radial velocity fields using the barycentre of the profile of the $\mathrm{H} \alpha$ line for each pixel with an accuracy for the central velocities of $\pm 5 \mathrm{~km} \mathrm{~s}^{-1}$. We masked the velocity field including only a rectangular area that contains the part of the radial velocity map corresponding to NGC 6090. Then, we superimposed the radial velocity profiles on the resulting velocity field and masked the radial velocity field based on the signal-to-noise ratio of each pixel, whose values were in the range from 24 in the centre of the galaxy system down to 4 in the most external pixels.

In addition to the data cube, we obtained a direct $\mathrm{H} \alpha$ image of NGC 6090 having the FP out of the optical path and with the same filter, with an exposure time of 90 seconds.

\section{MONOCHROMATIC AND CONTINUUM IMAGES}

On the DSS optical image of NGC 6090 (upper panel of Figure 1) we superimposed the direct $\mathrm{H} \alpha$ image obtained with PUMA. The ionized gas emitting at $\mathrm{H} \alpha$ that we detected was found within the central area of the object, which has 20.32 arcsec per side, i.e. 16 pixels per side, where $1 \mathrm{pix}=1.27 \operatorname{arcsec}=$ $0.726 \mathrm{kpc}$. The mean heliocentric radial velocity that we determined for the galactic system NGC 6090 is $8885 \mathrm{~km} \mathrm{~s}^{-1}$. We did not detect ionized gas emitting in $\mathrm{H} \alpha$ in the tidal tails (antennae), so we limited our kinematic analysis to the central area of NGC 6090 disregarding the antennae. In the bottom panel of Figure 1 we show the NICMOS/HST $2.22 \mu$ m image whose 22 arcsec field of view matches our results.

To visualize the morphology of NGC 6090 that the PUMA data show the contours of the $\mathrm{H} \alpha$ continuum (Figure 2 left panel) and monochromatic (Figure 2 right panel) emissions of NGC 6090 were superposed on the NICMOS/HST image.

The coordinates of the continuum maximum of NGC $6090 \mathrm{NE}$ are $\alpha_{\mathrm{J} 2000}=16^{\mathrm{h}} 11^{\mathrm{m}} 40.8^{\mathrm{s}}$, $\delta_{\mathrm{J} 2000}=+52^{\circ} 27^{\prime} 27^{\prime \prime} \cdot 32$, and the maximum $\mathrm{H} \alpha$ monochromatic emission occurs at $\alpha_{\mathrm{J} 2000}=$ $16^{\mathrm{h}} 11^{\mathrm{m}} 40.8^{\mathrm{s}}, \quad \delta_{\mathrm{J} 2000}=+52^{\circ} 27^{\prime} 27^{\prime \prime} \cdot 0 . \quad$ For NGC 6090 NE we can observe that the ionized gas emitting in $\mathrm{H} \alpha$ has a greater presence towards the west, i.e. towards its companion galaxy. But the isophotes of the continuum show that the stellar population has a greater presence towards the east. In addition, we note that the isophotes of the continuum are symmetrical with respect to the photometric position angle (PA), which is the angle of the major axis of the continuum with respect to the north in a counter-clockwise direction and whose value is $\simeq 0^{\circ}$. The pattern of the isophotes suggests the likely presence of a stellar bar.

For the galaxy NGC6090 SW, the coordinates of the photometric maximum are $\alpha_{\mathrm{J} 2000}=$ $16^{\mathrm{h}} 11^{\mathrm{m}} 40.4^{\mathrm{s}}, \delta_{\mathrm{J} 2000}=+52^{\circ} 27^{\prime} 22^{\prime \prime} \cdot 21$ and the coordinates of the $\mathrm{H} \alpha$ monochromatic maximum are $\alpha_{\text {J } 2000}=16^{\mathrm{h}} 11^{\mathrm{m}} 43.3^{\mathrm{s}}, \delta_{\mathrm{J} 2000}=+52^{\circ} 27^{\prime} 23^{\prime \prime}$.0. For this galaxy, we note that the continuum has a greater presence towards the south, the opposite side of its companion, and also that it is accumulated towards the opposite direction of its $\mathrm{H} \alpha$ maximum emission. Moreover, we note that the $\mathrm{H} \alpha$ monochromatic maximum is nearer to the the knot described in the literature as the maximum of the $2.22 \mu \mathrm{m}$ image (e.g. Dinshaw et al. 1999) than the photometric centre of the galaxy; this has created controversy in the literature about the position of the nuclei of NGC 6090 SW.

\section{KINEMATIC RESULTS}

\subsection{Velocity Fields}

In the previous section it was shown that the ionized gas emitting at $\mathrm{H} \alpha$ does not have the same morphology as the continuum emission. As a consequence of this phenomenon, due to the interaction between the galaxies, we do not expect that all the photometric and kinematic parameters will exactly match. In Figure 3, which shows the velocity field of NGC 6090 (with its isovelocities contour curves and the $2.22 \mu \mathrm{m}$ image superposed) it is possible to notice the velocity field of two independent galaxies, i.e. NGC 6090 presents two velocity fields ordered roughly on each one of its photometric maxima. Also, each velocity field has distortions towards the outside, mainly toward the northern side of both galaxies.

For NGC $6090 \mathrm{NE}$, the values of the radial velocities within its ordered velocity field, which reaches a radius of $R=5.6$ arcsec, lie in the range from 8783 to $8919 \mathrm{~km} \mathrm{~s}^{-1}$. For NGC $6090 \mathrm{SW}$, the radial velocities lie in the range from 8799 to $9143 \mathrm{~km} \mathrm{~s}^{-1}$ within its velocity field ordered up to a radius of $R=4.5$ arcsec. Furthermore, Figure 3 for NGC 6090 NE, shows that the isovelocity contour curves of the disc of the galaxy are not symmetric with respect to the kine- 

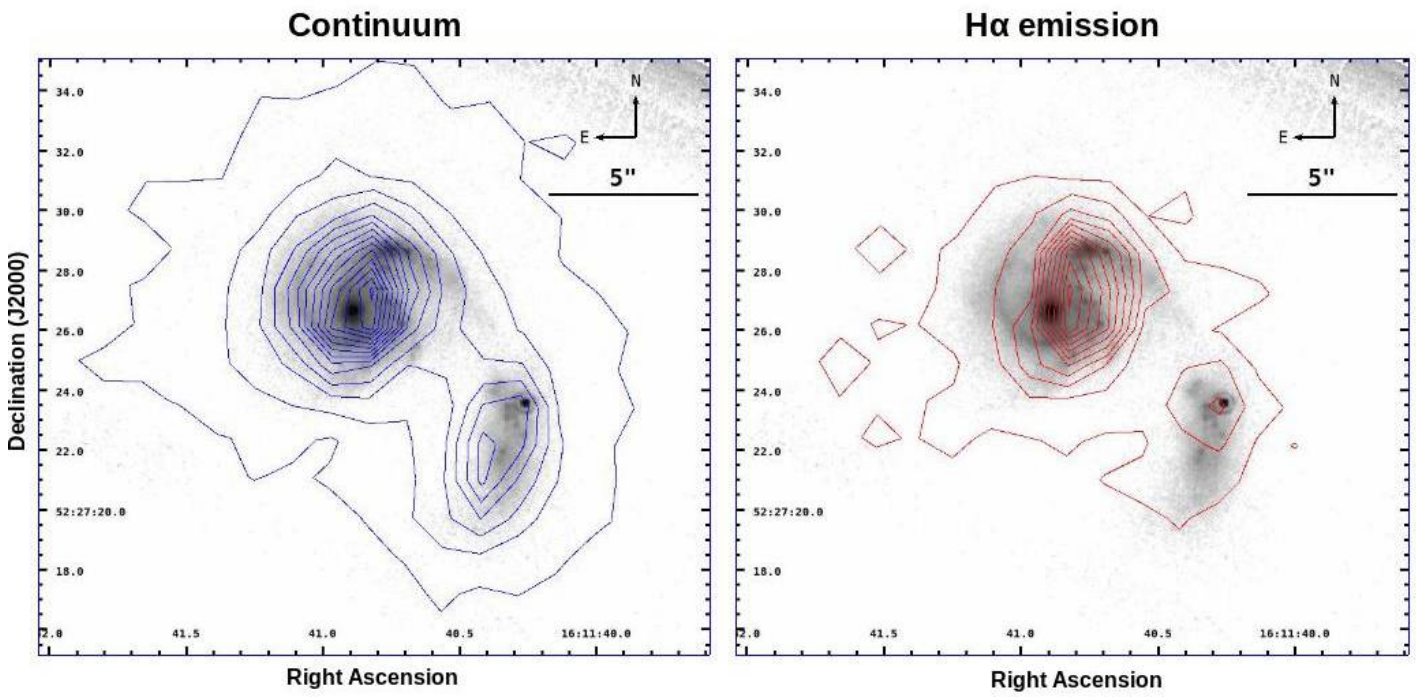

Fig. 2. Left: contours of the continuum linearly spaced from 1500 to 10000 ADU obtained from the H $\alpha$ data cube by the scanning FP interferometer, PUMA, overlaid on the NICMOS/HST $2.22 \mu \mathrm{m}$ image in a 22 arcsec field of view centred on NGC 6090. Right: contours of the $\mathrm{H} \alpha$ monochromatic image linearly spaced from 4000 to 240000 ADU obtained with PUMA, overlaid on the NICMOS/HST $2.22 \mu \mathrm{m}$ image in a 22 arcsec field of view centred on NGC 6090. The color figure can be viewed online.

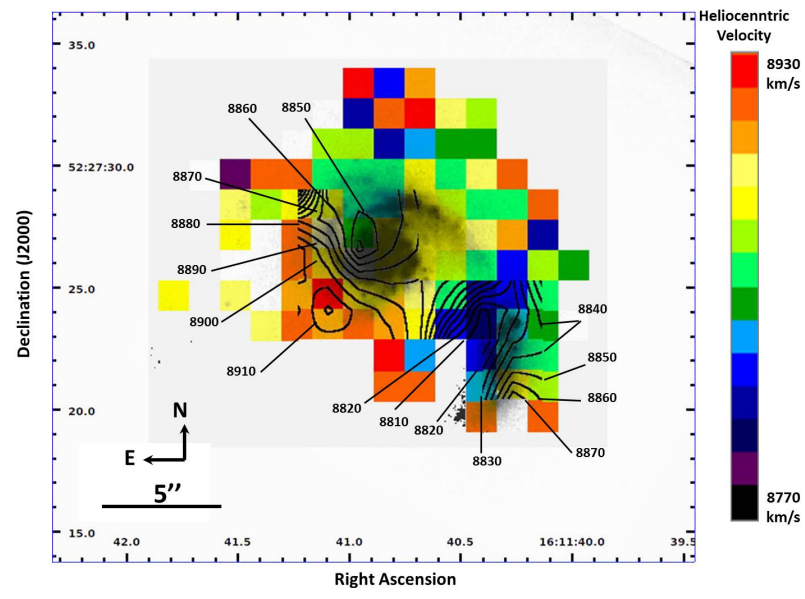

Fig. 3. Velocity field acquired with the $\mathrm{H} \alpha$ data cube observation obtained with the scanning FP interferometer, PUMA, in a 26 arcsec field of view centred in NGC 6090. On the velocity field are overlaid, firstly, the NICMOS/HST $2.22 \mu \mathrm{m}$ image and, secondly, the isovelocity contours of each galaxy which are linearly spaced by a factor of 10 starting from $8810 \mathrm{~km} \mathrm{~s}^{-1}$. Each isovelocity is labelled in $\mathrm{km} \mathrm{s}^{-1}$ units. The color figure can be viewed online.

matic minor axis. This feature, added to the morphology indicated by the continuum contours described in the previous section, suggests the pres- ence of a stellar bar, in agreement with the criteria proposed by Bosma (1981).

\subsection{Rotation Curves}

From the morphological and kinematic features described in previous sections, we can argue that we have detected two independent galaxies inside the NGC 6090 galactic system. In this kind of interacting systems the radial motions, such as inflows/outflows or motions perpendicular to the galactic disc, are second order effects, otherwise the discs would be destroyed. We started studying the circular motion of each galaxy in NGC 6090, which corresponds to the most basic motion that can be assumed in a disc galaxy. In this way, the rotation curve of each galaxy was obtained from the kinematic data acquired with our FP observations using the $\mathrm{ADHOCw}$ software, which computes the radial velocity of the galaxies in every single pixel using the bary centre of the $\mathrm{H} \alpha$ profile observed in each pixel.

In doing so, we use the geometric and kinematic assumptions proposed by Mihalas \& Binney (1981). Supposing that each galaxy has a well-defined disc and that the system rotates about an axis that is perpendicular to the galactic plane which is inclined an angle $i$ to the plane of the sky; we use the polar coordinate systems $(R, \theta)$ in the plane of the galaxy 

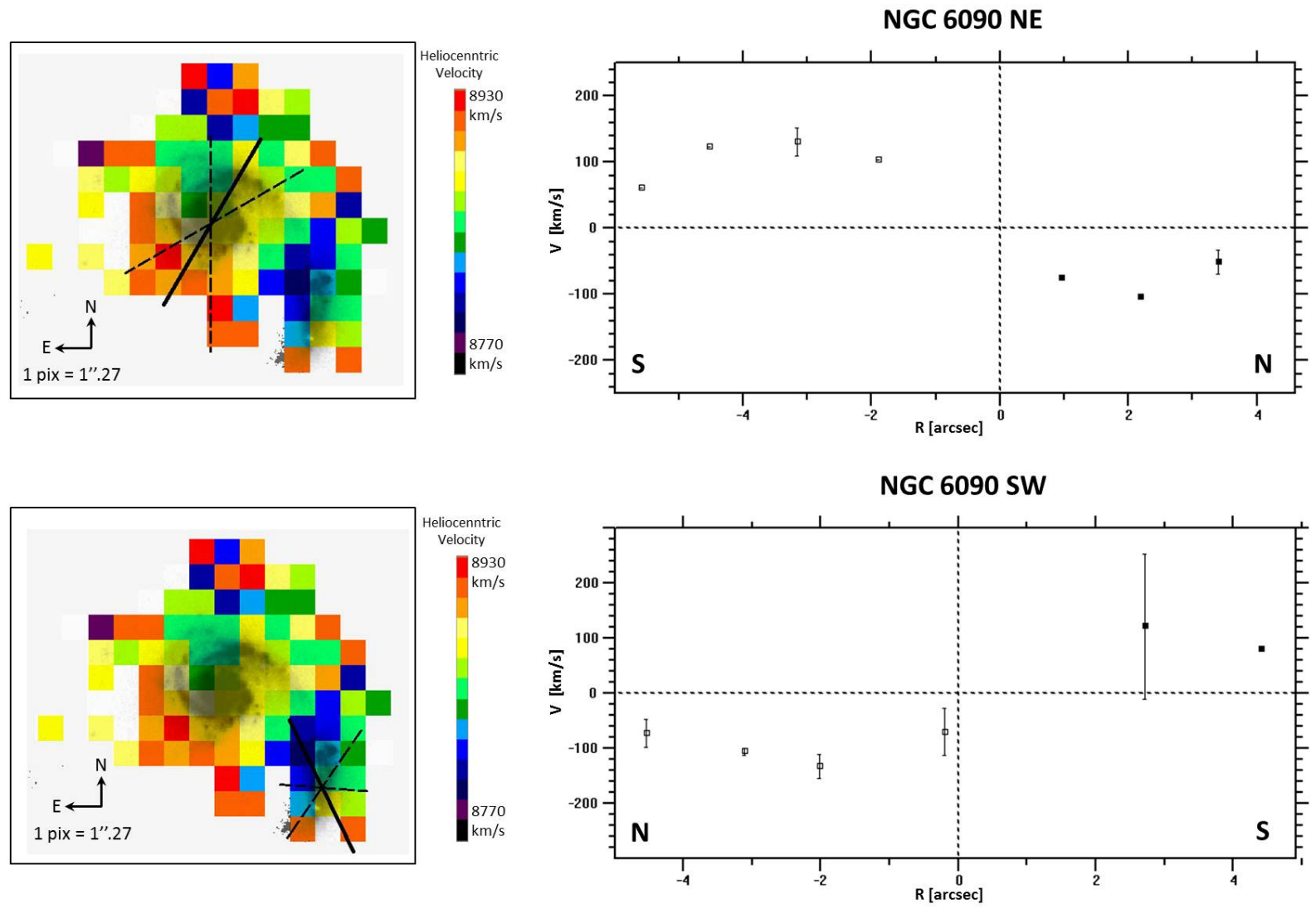

Fig. 4. Left panels show the velocity maps acquired with the $\mathrm{H} \alpha$ data cube obtained with the scanning FP interferometer, PUMA, in a 22 arcsec field of view. On these maps was superposed the NICMOS/HST $2.22 \mu \mathrm{m}$ image as a frame of reference for the position of galaxy components in the galactic pair; then, on each map, the solid line represents the major kinematic axis which indicates the kinematic $P A$, and the dashed lines represent the sector angle that was considered for the computation of the rotation curve of each galaxy: NGC $6090 \mathrm{NE}$ above and NGC $6090 \mathrm{SW}$ below. In the right panels are shown the rotation curves of each galaxy, obtained up to where the velocity field is ordered for each galaxy; the dispersion associated with the averaged value of pixels within the same sector is indicated with bars. The color figure can be viewed online.

and $(\rho, \phi)$ in the plane of the sky, locating the origin at the point where the rotation axis lies in the plane of the sky; the radial velocity $\left(V_{\text {obs }}\right)$ measured at $(\rho, \phi)$ is $V_{\text {obs }}(\rho, \phi)=V_{\text {sys }}+V_{r}(R, \theta) \sin \theta \sin i+$ $V_{\Theta}(R, \theta) \cos \theta \sin i+V_{z}(R, \theta) \cos i$, where $R^{2}=$ $\rho^{2}\left(\cos ^{2} \phi+\sec ^{2} i \sin ^{2} \phi\right)$ and $\tan \phi=\sec i \tan \theta$, with $V_{\text {sys }}$ the mean heliocentric radial velocity of the system as a whole (that is, of its centre of mass); $V_{r}$ and $V_{\Theta}$ are the radial and tangential velocities in the plane, respectively, and $V_{z}$ is the velocity perpendicular to the plane. Given that rotation is generally the dominant form of motion in disc galaxies, we assume $V_{r}$ and $V_{z}$ equal to zero. Moreover, under the assumption that there is axial symmetry about the centre of galaxy, $V_{\Theta}$ will depend only on $R$. Thus, the tangential velocity in the plane of the galaxy $\left(V_{\Theta}\right)$ was obtained with the radial velocity $V_{o b s_{k}}$ of the $k$ th region on the galaxy in terms of observable quantities:

$$
V_{\Theta}\left(R_{k}\right)=\frac{V_{o b s_{k}}-V_{s y s}}{\cos \theta_{k} \sin i},
$$

where $R_{k}$ and $\theta_{k}$ are given in terms of the coordinates $\rho_{k}$ and $\phi_{k}$ of the $k$ th galaxy region on the sky which corresponds to the $k$ th pixel of the velocity field that was obtained with our FP observations.

The $\mathrm{ADHOCw}$ software averages the measured velocity of the pixels that are at the same distance from the kinematic centre along the major axis, indicating the dispersion associated to this average with bars on the points of the rotational observed curves. In order to avoid strong dispersions associated to the points of the rotation curves the pixels within an angular sector along the kinematic major axis 
TABLE 3

ROTATION CURVE PARAMETERS AND MASS RANGE OF EACH GALAXY IN THE NGC6090 PAIR

\begin{tabular}{|c|c|c|}
\hline Parameter & NGC $6090 \mathrm{NE}$ & NGC 6090 SW \\
\hline \multirow[t]{2}{*}{ Coordinates (J2000) } & $\alpha=16^{\mathrm{h}} 11^{\mathrm{m}} 40.84^{\mathrm{s}}$ & $\alpha=16^{\mathrm{h}} 11^{\mathrm{m}} 40.42^{\mathrm{s}}$ \\
\hline & $\delta=+52^{\circ} 27^{\prime} 26^{\prime \prime} .94$ & $\delta=+52^{\circ} \quad 27^{\prime} \quad 22^{\prime \prime} \cdot 21$ \\
\hline Distance $(\mathrm{Mpc})$ & 118 & 118 \\
\hline Systemic velocity $\left(\mathrm{km} \mathrm{s}^{-1}\right)$ & 8880 & 8890 \\
\hline Max. rotation velocity $\left(\mathrm{km} \mathrm{s}^{-1}\right)$ & $130 \pm 5$ & $132 \pm 5$ \\
\hline Kinematic $P A\left(^{\circ}\right)$ & $150 \pm 3$ & $26 \pm 3$ \\
\hline Inclination $\left(^{\circ}\right)$ & $13.9 \pm 0.5$ & $64.8 \pm 0.5$ \\
\hline Radius (arcsec) & 5.6 & 4.5 \\
\hline Radius (kpc) & 3.2 & 2.6 \\
\hline $\operatorname{Mass}\left(M_{\odot}\right)$ & $0.76 \times 10^{10}$ to $1.26 \times 10^{10}$ & $0.63 \times 10^{10}$ to $1.05 \times 10^{10}$ \\
\hline
\end{tabular}

were considered (e.g. Fuentes-Carrera et al. 2004, 2007; Repetto et al. 2010) as shown in left panels of Figure 4. Then, assuming that each galaxy has a well-defined disc, due to its inclination it would look like an ellipse on the sky plane with apparent axis lengths $a$ and $b$; so, its inclination can be computed by $i=\cos ^{-1}(b / a)$. We applied the ELLIPSE task of IRAF to the $2.22 \mu \mathrm{m}$ image to estimate the inclination of the galaxies from the isophotal ellipses traced on these images. Finally, we considered that the systemic velocity of each galaxy is in agreement with the radial velocity of its own kinematic centre.

Table 3 contains the parameters used to calculate the rotation curve of each galaxy. The listed values correspond to those values with which we obtained the rotation curves from our observations; changes in these values would result in scattered points in the rotation curves instead of symmetric rotation curves.

The rotation curve of NGC 6090 NE presented in the upper right panel of Figure 4 was obtained with pixels in the velocity field within an angular sector of $30^{\circ}$ around the kinematic major axis of the galaxy as marked in the upper left panel of Figure 4. The physical coordinates of the kinematic centre, derived as the position around the photometric centre at which the scatter in rotation curve is minimized, are $\alpha_{\mathrm{J} 2000}=16^{\mathrm{h}} 11^{\mathrm{m}} 40.84^{\mathrm{s}}, \delta_{\mathrm{J} 2000}=+52^{\circ} 27^{\prime} 26^{\prime \prime} 94$. The kinematic centre used to compute the rotation curve in this galaxy matches the photometric centre within 0.4 arcsec. Using ADHOCw software, the kinematic parameters that give us the most symmetric, smooth and least scattered curve inside of the radius $R=5.6 \operatorname{arcsec}$ are $P A=150^{\circ} \pm 3$, $i=13.9^{\circ} \pm 0.5$, and $V_{\mathrm{sys}}=8880 \mathrm{~km} \mathrm{~s}^{-1}$. We determined that the heliocentric distance to this galaxy is $118 \mathrm{Mpc}$ through the Hubble law (Hubble 1936). The rotation curve shows that the north of the galaxy is blue-shifted, i.e. in the north side of NGC $6090 \mathrm{NE}$ the gas approaches us, while in the south the gas moves away. Its maximum rotational velocity reaches $V_{\Theta \max }=130 \pm 5 \mathrm{~km} \mathrm{~s}^{-1}$ at $3 \operatorname{arcsec}$ to the south from its kinematic centre.

The rotation curve of NGC6090 SW (bottom right panel of Figure 4) was obtained with pixels in the velocity field within an angular sector of $60^{\circ}$ around the kinematic major axis of the galaxy. The physical coordinates of the kinematic centre are $\alpha_{\mathrm{J} 2000}=16^{\mathrm{h}} 11^{\mathrm{m}} 40.42^{\mathrm{s}}, \delta_{\mathrm{J} 2000}=+52^{\circ} 27^{\prime} 22^{\prime \prime} \cdot 21$. The kinematic centre used to compute the rotation curve in this galaxy matches the photometric centre within 0.77 arcsec. The kinematic parameters that reduce significantly the asymmetry and scatter in the rotation curve inside of radius $R=4.5$ arcsec were, in this case, $P A=26^{\circ} \pm 3, i=64.8^{\circ} \pm 0.5$, and $V_{\text {sys }}=8890 \mathrm{~km} \mathrm{~s}^{-1}$. Just as we calculated the heliocentric distance for NGC $6090 \mathrm{NE}$, we have obtained that the heliocentric distance of NGC $6090 \mathrm{SW}$ is $118 \mathrm{Mpc}$. The rotation curve of NGC $6090 \mathrm{SW}$ shows that the north side is blue-shifted as well as the northeastern galaxy. The maximum rotational velocity of NGC $6090 \mathrm{SW}$ is $V_{\Theta \max }=132 \pm 5 \mathrm{~km} \mathrm{~s}^{-1}$ at 2 arcsec to the north from its kinematic centre.

In the rotation curve of NGC $6090 \mathrm{SW}$ there is a point on the receding side with a high dispersion associated to the average of the radial velocities computed using only two pixels of the velocity field; the pixel on the kinematic centre considered the framework to draw the radial velocities in the rotation curve and hence, considered the point with the lowest radial velocity value, and the pixel at the south- 


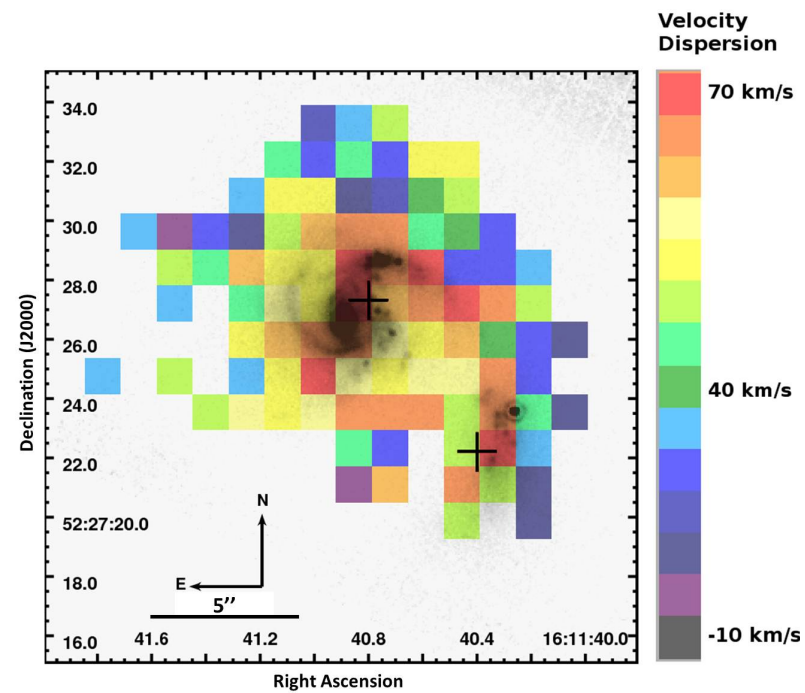

Fig. 5. Velocity dispersion map obtained using the PUMA in a 22 arcsec field of view centred on NGC 6090 superposed on the NICMOS/HST $2.22 \mu \mathrm{m}$ image. Symbols '+' represent the photometric centre of each galaxy. The color figure can be viewed online.

west of the kinematic centre with the highest radial velocity value of the velocity field of the entire system. Despite that, we decided to use this point only to get a better view of the rotation curve of this galaxy toward the south.

\subsection{Velocity Dispersion Field}

Through the full width at half maximum (FWHM) of Gaussian functions fitted to the velocity profile of the $\mathrm{H} \alpha$ line in each pixel we obtained the velocity dispersion map of the galaxy system. Figure 5 displays the velocity dispersion map of NGC 6090 with the NICMOS/HST image overlaid. In NGC 6090 NE the velocity dispersion increases gradually from $\approx 45-55 \mathrm{~km} \mathrm{~s}^{-1}$ in the eastern side of the galaxy, to $59-66 \mathrm{~km} \mathrm{~s}^{-1}$ in the centre, and then up to $75 \mathrm{~km} \mathrm{~s}^{-1}$ in its north-western spiral arm near to what might look like a bridge between the galaxies. In NGC $6090 \mathrm{SW}$, the velocity dispersion maximum is $85 \mathrm{~km} \mathrm{~s}^{-1}$ next to the position of its photometric centre. Then, in its surroundings the velocity dispersion range is $\approx 40 \mathrm{~km} \mathrm{~s}^{-1}$ increasing to the north, where it is $\approx 69 \mathrm{~km} \mathrm{~s}^{-1}$.

In the north side of the galaxy system and between both galaxies, broader profiles were noticed through double radial velocity profiles with a low signal to noise ratio, detected with PUMA and mainly

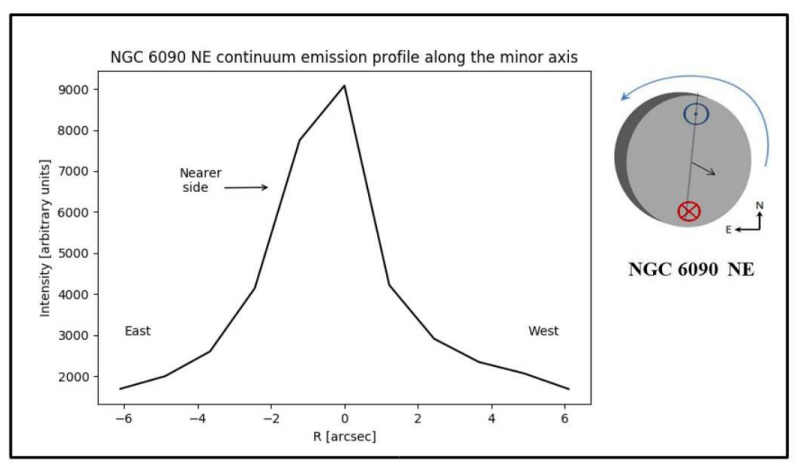

Fig. 6. Intensity profile of the continuum map of NGC 6090 NE along the minor axis centred on the galactic phometric centre. Following the gradient criterion of Pasha (1985), the far side of the galaxy would be the one that decreases more smoothly. Therefore, the nearest side to the observer of NGC 6090 is the eastern one. The top-right panel shows a schematic 3D orientation of NGC 6090 NE derived from our kinematic analysis. The color figure can be viewed online.

located in the bridge zone. Those broader profiles were already detected by Cortijo-Ferrero et al. (2017) and interpreted as a consequence of shocks in earlystage mergers. This feature of the velocity dispersion field indicates that NGC 6090 has an interaction bridge between its galaxies. Those pixels with double velocity profiles are outside of the ordered velocity field of each galaxy, so that they do not contribute at any point in the computation of the rotation curves. Moreover, given their low signal to noise ratio we do not consider them in this work.

\subsection{The Rotation Sense of NGC $6090 \mathrm{NE}$}

Radial-velocity measurements cannot by themselves distinguish leading and trailing spirals in thin disks. To determine whether a given galaxy leads or trails, we must determine which side of the galaxy is closer to us. Thus, to determine if the galaxies in NGC 6090 lead or trail, we followed the gradient criterion of Pasha (1985): the apparent brightness of the nuclear region falls off unequally from the centre outward along the minor axis, the far side being the one where the surface-brightness profile declines more smoothly. This means that if the inner disc is dusty, it absorbs a significant fraction of the starlight passing through it, then the surface brightness of the bulge at a given distance along its apparent minor axis will be lower on the near side (Binney \& Tremaine 1987). In this way, for each galaxy in the 
NGC 6090 pair the intensity profile of the continuum emission was obtained along their minor axis in order to determine the rotation sense of each one.

For NGC $6090 \mathrm{NE}$, we extracted the intensity profile of the continuum from its minor photometric axis (see Figure 2 in $\S 3$ ), as shown in Figure 6. This profile indicates that the side nearest to the observer is the eastern side of the galaxy. Then, considering that the rotation curve indicates that the gas on the northern side is blue-shifted and, availing us of the dust lanes which trace the arms structure of this galaxy (see right panel of Figure 1), we can infer that this galaxy has its arms rotating in a trailing direction.

On the other hand, for NGC $6090 \mathrm{SW}$ it was not possible to obtain an accurate result through which we might determine its rotation sense, due to the low number of pixels with continuum emission along its minor axis. In addition, the image of $2.22 \mu \mathrm{m}$ does not clearly mark the structure of spiral arms.

\section{MASS ESTIMATES USING DYNAMICAL ANALYSIS}

A range of possible values for the mass of each galaxy in the NGC 6090 system was calculated using the approach described by Lequeux (1983), as has been done before when studying pairs of galaxies (e.g. Amram et al. 1992; Fuentes-Carrera et al. 2007; Repetto et al. 2010). This approach consists in calculating the mass $M(R)$ up to a certain radius where the rotation velocity $V(R)$ has been measured. The method considers two extreme cases to evaluate the mass of galaxies: the galaxy as a flat disc or the galaxy as a spherical system. Thus, the mass of the spiral galaxies is in the range of $M(R)=\kappa R V^{2}(R) / G$, where $G$ is the gravitational constant and the coefficient $\kappa$ is a constant in the range $(0.6,1.0)$, whose limiting values correspond to the galaxy being dominated by a flat disc or by a massive spherical halo, respectively.

Thus, from the NGC6090 NE rotation curve, the maximum rotation velocity of this galaxy is $V_{N E}=130 \mathrm{~km} \mathrm{~s}^{-1}$ which is reached at a radius of $R=2.47 \mathrm{arcsec}$. For this galaxy the mass range obtained is $M_{N E}=1.26 \kappa \times 10^{10} M_{\odot}$. From the NGC $6090 \mathrm{SW}$ rotation curve, the maximum rotation velocity is $V_{S W}=132 \mathrm{~km} \mathrm{~s}^{-1}$ reached at $R=1.97$ arcsec. For this galaxy the mass range is $M_{S W}=1.05 \kappa \times 10^{10} M_{\odot}$. Therefore, the sum of both masses when both galaxies are dominated by a massive spherical halo is $M_{s p h}=2.31 \times 10^{10} M_{\odot}$, and when both galaxies are dominated by flat disc, the mass is $M_{\text {flat }}=1.39 \times 10^{10} M_{\odot}$.
When two galaxies of roughly equal mass have an encounter, their extended halos may merge to form a common halo. Then, the galaxies are expected to orbit in a common halo until dynamical friction and tidal interactions remove sufficient orbital energy for the galaxies to merge (e.g. Binney \& Tremaine 1987; Mo et al. 2010). Thus, due to the active state of interaction of the galaxies in NGC 6090 it is possible to infer that they are dominated by a flat disc individually, but a further study on the dynamics of this encounter would be necessary to dismiss the case in which both galaxies are dominated by a spherical halo.

A second independent way of obtaining the mass value of a pair of galaxies is the method proposed by Karachentsev (1987), which calculates the mass from the relative orbital motion of the components. This method consists of assuming that the components of the pair move in a circular orbit with a velocity $V_{12}$ and a spatial separation $r$. When $V_{12}$ and $r$ are transformed into observable quantities, they become the difference of the velocity projected in the line of sight, $y=V_{12} \sin i \cos \Omega$, and the separation projected on the sky plane, $X=r\left(1-\sin ^{2} i \sin ^{2} \Omega\right)^{1 / 2}$, where $i$ is the angle between the plane of the orbit and the plane of the sky, and $\Omega$ the angle between the line of sight and the line connecting both galaxies of the pair. For circular motion, Karachentsev (1987) obtained the projection factor $\langle\eta\rangle=3 \pi / 32$. Therefore, the total mass of the pair is:

$$
M=\frac{32}{3 \pi} \frac{X y^{2}}{G},
$$

where $G$ is the gravitational constant. As $y$ and $X$ depend on a sinusoidal function, in order to obtain the upper limit of mass with this method without loss of generality, we assumed that the orbital plane has an inclination of $i=90^{\circ}$. Then, the angle between the line of sight and the line connecting the pair is $\Omega=\sin ^{-1}(L / D)$, where $L=4.1 \mathrm{kpc}$ is the distance of the nuclei projected on the sky plane and $D=133.1 \mathrm{kpc}$ is the size of the line connecting the galaxies, which results in $\Omega=1.76^{\circ}$. Thus, the velocity projected on the line of sight and the separation projected on the sky plane is $y \approx V_{12}$ and $X \approx r$. Assuming a circular orbit, the total mass would be:

$$
M_{\text {orbital }}=\frac{32}{3 \pi} \frac{r V_{12}^{2}}{G},
$$

where $V_{12}$ is the difference between the velocities of the galaxies, and $r$ is the projected separation between the nucleus of each galaxy. For the system NGC 6090 we have, $V_{12}=10 \mathrm{~km} \mathrm{~s}^{-1}$ 
and $r=4.1 \mathrm{kpc}$. Therefore, the orbital mass is $M_{\text {orbital }}=3.23 \times 10^{8} M_{\odot}$, which is two orders of magnitude smaller than the sum of the individual masses obtained with the method proposed by Lequeux (1983). We think that this low value of the mass is due to the fact that the projected velocity difference $V_{12}$ is quite small.

\section{DISCUSSION}

At optical wavelengths, NGC 6090 appears as a double nuclei system with two curved antennas (tidal tails), so that this galaxy system has been described as similar to NGC 4038/39 (the Antennae) (e.g. Toomre \& Toomre 1972; Martin et al. 1991; Mazzarella \& Boroson 1993; Dinshaw et al. 1999; Bryant \& Scoville 1999). With the goal of studying kinematic similarities between both systems produced by the interaction of their own galaxies, we studied the $\mathrm{H} \alpha$ kinematics of NGC 6090 using the scanning Fabry-Perot interferometer, PUMA. The $\mathrm{H} \alpha$ kinematics of the galaxy pair the Antennae has been studied through observations obtained with another FP by Amram et al. (1992). In neither galaxy system, NGC 6090 and the Antennae, was emission of ionized hydrogen detected in their tidal tails, i.e. in both galaxy systems ionized gas emitting at $\mathrm{H} \alpha$ was only detected in the central zone. Therefore, Amram et al. (1992) performed a kinematic analysis of the disc of the Antennae, as we have done for NGC 6090 in this paper.

Amram et al. (1992) reported that in the Antennae, ionized gas emitting at $\mathrm{H} \alpha$ is deficient compared with its continuum emission, but that the emissions tend to grow toward its part farthest from its companion. Then, the Antennae nuclei matched unambiguously with observations at $2.2 \mu \mathrm{m}$. The velocity field is not ordered throughout the system; none of its component galaxies show the behaviour of a rotating disc. Additionally, there were no detected pixels with double velocity profiles. Finally, using the Lequeux (1983) method to measure the mass of the galaxies, Amram et al. (1992) found that the individual masses of the galaxies in the Antennae are approximately $2 \times 10^{10} M_{\odot}$.

For NGC 6090, in $\S 3$ we noted that the intensity of ionized gas emitting in $\mathrm{H} \alpha$ is lower with respect to that of the continuum; but within each galaxy, the ionized hydrogen gas is situated near to its companion, while the old stellar population is lagging in the opposite direction. The positions of the nuclei reported at $2.22 \mu \mathrm{m}$ by Dinshaw et al. (1999), the HI continuum maxima reported by Condon et al.

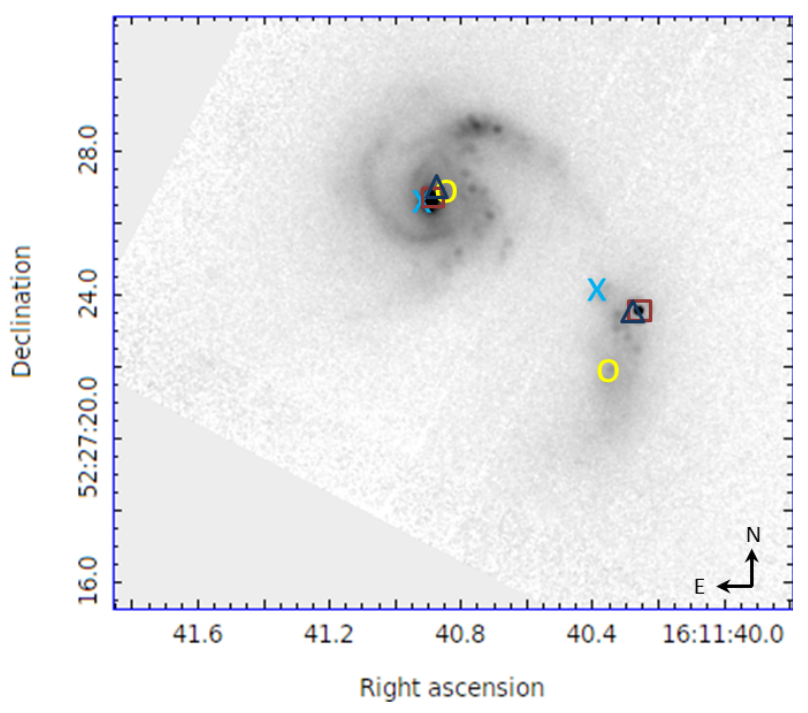

Fig. 7. NICMOS/HST image at $2.22 \mu \mathrm{m}$ of a field of view of 16 arcsec centered at NGC 6090. In this image we have marked the positions of the maximum emissions of the continuum $(\mathrm{O})$, the $\mathrm{H} \alpha$ monochromatic emission $(\triangle)$, the radio continuum (X) and the infrared $(\square)$. The color figure can be viewed online.

(1990), and the monochromatic and continuum $\mathrm{H} \alpha$ maxima that we obtained in this paper are marked on the NICMOS/HST image in Figure 7.

For NGC 6090 NE, we found that the maxima of the monochromatic and continuum of the PUMA observations almost completely coincide and match the kinematic centre (within $0.4 \operatorname{arcsec}$ ). In Figure 7 we show that the differences between the position of the nucleus of this galaxy reported in the literature and our results are minimal.

However, for the galaxy NGC 6090 SW the maxima of the continuum and the monochromatic emissions derived from our observations of the ionized gas emitting in $\mathrm{H} \alpha$ are not nearly in the same position as those in NGC 6090 NE. This galaxy presents greater emissions of ionized hydrogen towards the north than the south, so that the photometric maximum is located to the south, while the maximum monochromatic emission is in the north. Thus, for NGC $6090 \mathrm{SW}$, the position of the monochromatic emission maximum is $\alpha_{\mathrm{J} 2000_{\mathrm{H} \alpha}}=16^{\mathrm{h}} 11^{\mathrm{m}} 40.3^{\mathrm{s}}$, $\delta_{\mathrm{J}^{2} 000_{\mathrm{H} \alpha}}=+52^{\circ} 27^{\prime} 23^{\prime \prime}$, , and the maximum of the continuum is $\alpha_{\mathrm{J} 2000_{\text {cont }}}=16^{\mathrm{h}} 11^{\mathrm{m}} 40.4^{\mathrm{s}}, \delta_{\mathrm{J} 2000_{\text {cont }}}=$ $+52^{\circ} 27^{\prime} 22^{\prime \prime}$, which match the kinematic centre and the velocity dispersion maximum. The maximum of the $2.22 \mu \mathrm{m}$ emission is at $\alpha_{\mathrm{J} 2000}=16^{\mathrm{h}} 11^{\mathrm{m}} 40.3^{\mathrm{s}}$, $\delta_{\mathrm{J} 2000}=+52^{\circ} 27^{\prime} 23^{\prime \prime}$ ( (Dinshaw et al. 1999), and 
TABLE 4

\section{MASSES OF THE GALAXY SYSTEM NGC 6090 FOUND IN THE LITERATURE AT DIFFERENT WAVELENGTHS COMPARED WITH THE ONES FOUND IN THIS WORK}

\begin{tabular}{lcc}
\hline Author & $\begin{array}{c}\text { Phase of the } \\
\text { IM observed }\end{array}$ & $\begin{array}{c}\text { Mass } \\
{\left[M_{\odot} \times 10^{10}\right]}\end{array}$ \\
\hline Sanders et al. (1991) & $\mathrm{H}_{2}$ & 1.4 \\
Bryant \& Scoville (1999) & $\mathrm{H}_{2}$ (dyn) & 0.46 \\
& $\mathrm{H}_{2 \text { (gas) }}$ & 2.29 \\
& $\mathrm{HI}$ & 3.9 \\
Hummel et al. (1987) & $\mathrm{MIR}$ & 5.02 \\
Chisholm et al. (2016a) & $\mathrm{MIR}$ & 4.2 \\
Cortijo-Ferrero et al. (2017) & $3650-6950 \AA$ & 6.8 \\
Cortijo-Ferrero et al. (2017) & $\mathrm{H} \alpha_{\text {sum flat disc }}$ & 1.39 \\
This work & $\mathrm{H} \alpha_{\text {sum sph halo }}$ & 2.31 \\
& $\mathrm{H} \alpha_{\text {orbital }}$ & 0.032 \\
\hline
\end{tabular}

the maximum radio continuum is at $\alpha_{\mathrm{J} 2000}=$ $16^{\mathrm{h}} 11^{\mathrm{m}} 40.8^{\mathrm{s}}, \delta_{\mathrm{J} 2000}=+52^{\circ} 27^{\prime} 27^{\prime \prime}$ (Hummel et al. 1987; Condon et al. 1990).

Dinshaw et al. (1999) compared the position of the brightest point in the $2.22 \mu \mathrm{m}$ image with the maximum of the radio-continuum of this galaxy; they proposed that the infrared peak could be a field star. However, the emission of a field star could not be detected as the maximum of the redshifted $\mathrm{H} \alpha$ line of our observations of NGC 6090. Therefore, it may be concluded that the infrared maximum for the southwestern galaxy is a component itself. This possibility is supported by Cortijo-Ferrero et al. (2017), who argued that the main absorption lines of their observations in the range from 3700 to $7100 \AA$ at the knot of $2.22 \mu \mathrm{m}$ have the same redshift as the galaxy. However, Cortijo-Ferrero et al. (2017) obtained that the position of the continuum maximum is southward of the infrared peak, near where we detected the continuum maximum, the kinematic centre and the velocity dispersion maximum of NGC $6090 \mathrm{SW}$. Thus, taking the maximum of the $\mathrm{H} \alpha$ continuum as the nucleus of the southwest galaxy, we determined that the separation of the nuclei of the two members of NGC 6090 is 7.11 arcsec. Assuming that the average distance of the galaxy system NGC 6090 is $118 \mathrm{Mpc}$, the distance between its nuclei is $4.1 \mathrm{kpc}$.

Regarding the velocity field of NGC 6090, we said in $\S 4.1$ that this galaxy system has two ordered velocity fields which allowed us to compute the rotation curves of each galaxy in the NGC 6090 system. The rotation curves have maximum rotational velocity larger than the dispersion or residual velocity fields: the northeast galaxy has a maximum rotational velocity $V_{N E}=130 \mathrm{kms}^{-1}$ and its velocity dispersion is in the range $\approx 45-66 \mathrm{~km} \mathrm{~s}^{-1}$ inside of its disc; meanwhile NGC $6090 \mathrm{SW}$ has a maximum rotational velocity $V_{S W}=132 \mathrm{~km} \mathrm{~s}^{-1}$, with its velocity dispersion in the range $\approx 40-69 \mathrm{~km} \mathrm{~s}^{-1}$ inside the disc. Both galaxies reach their maximum velocity dispersion outside the disc, but even these values were less than their maxima rotational velocities.

Furthermore, with the continumm map obtained from our observations, with the asymmetric isovelocities contour curves, and the non-circular motions that we detected in NGC $6090 \mathrm{NE}$, we confirmed the assumption about the presence of a bar structure in this galaxy made in the infrared by Dinshaw et al. (1999) (see figure 2 of their paper) and at optical wavelengths by Cortijo-Ferrero et al. (2017).

In their paper, Amram et al. (1992) concluded that the encounter of the galaxies that make up the Antennae is in an advanced stage of interaction. In the case of NGC 6090, the features that we have discussed about its two ordered velocity fields, continuum and monochromatic maps, and the behaviour of its velocity dispersion field that signals an interaction bridge between galaxies, allow us to conclude that NGC 6090 is a system in a less advanced stage of interaction than the Antennae, although both systems are in an interaction stage previous to a merger.

Regarding the mass values, Table 4 contains the values of the masses reported in the literature for the NGC 6090 system at different wavelengths compared to our results. The range of the sum of independent masses estimated in this paper from the rota- 
tion curves of each galaxy obtained with the method of Lequeux (1983) is of the same order of magnitude as the masses estimated by other authors in the infrared (Sanders et al. 1991; Bryant \& Scoville 1999; Chisholm et al. 2016a; Cortijo-Ferrero et al. 2017) and radio continuum (Martin et al. 1991), which were determined by means of the mass-to-light ratio. However, the mass of the NGC 6090 system calculated with the method proposed by Karachentsev (1987) is two orders of magnitude smaller than the former values, and an order of magnitude smaller than the dynamic mass estimated by Bryant \& Scoville (1999).

The sum of the masses computed from the maximum rotation velocity is of the same order of magnitude as the masses acquired from the mass-to-light ratio at other wavelengths. They also coincide with the value determined by Sanders et al. (1991) and Bryant \& Scoville (1999), both if each galaxy is dominated by a flat disc or a spherical halo. Hence, we can argue that the kinematic parameters that we obtained in this work are reliable. The difference of an order of magnitude in the orbital mass can be consequence to our geometrical assumptions of the movement of NGC $6090 \mathrm{SW}$ around of its companion.

Most of the numerical simulations have assumed similar initial conditions to model different features of the Antennae (e.g. Toomre \& Toomre 1972; Barnes 1988; Teyssier et al. 2010; Renaud et al. 2008, 2015), either its morphology or the formation of star clusters during the merger. They obtain always the same feature: two long tidal tails that form the antennae. Therefore, in general, the symmetry of the tidal action allows the formation of four arms if the two companions are disc galaxies; when the masses of the two interacting galaxies are equal or similar, the two internal spiral arms join up to form a bridge, while the two external arms are drawn into two antennae which remain for one or two billion years (Combes et al. 2002). This phenomenon is happening to the galaxies of NGC 6090, which have similar mass values. We note the two tidal tails and the bridge between them, well defined by the nortweastern arm of NGC $6090 \mathrm{NE}$.

Finally, Sugai et al. (2004) observed young starbursts occurring in regions offset from the galactic nuclei to the northwestern spiral arm of NGC 6090 NE. They inferred that if NGC $6090 \mathrm{NE}$ had its arms trailing, the molecular gas clouds were just finishing an interaction with the central molecular gas. In $\S 4.4$ we showed that the spiral arms in the north-eastern galaxy are trailing, favoring this theory. Another likely consequence of the trailing arms of NGC 6090 NE could be the outflow at the northeastern side of NGC $6090 \mathrm{SW}$ detected by Chisholm et al. (2016b) in the UV band (see Figure 1 of their paper), which coincides with a region of high $\mathrm{H} \alpha$ velocity dispersion in NGC $6090 \mathrm{SW}$, which could have been formed by ram pressure during the interaction.

\section{CONCLUSIONS}

In this paper we present our observations of the ionized gas emitting at $\mathrm{H} \alpha$ of the isolated pair of galaxies NGC 6090 (KPG 486) carried out with the scanning Fabry-Perot interferometer, PUMA. This emission was detected solely in the central area of the system. Through our observations we obtained the monochromatic emission (ionized gas emitting at $\mathrm{H} \alpha$ ), the continuum, the velocity field and the velocity dispersion maps. The velocity field of NGC 6090 shows two regions with ordered isovelocity contour curves from which we computed the rotation curve of each galaxy of the pair.

Using the rotation curves we obtained the maximum rotation velocity, the mass of each galaxy, and the mass of the galaxy system. The sum of the individual masses that we calculated is consistent with the masses found in the literature, obtained with the mass-to-light ratio for different wavelengths. In addition, we obtained the orbital mass of NGC 6090, which differs by two orders of magnitude from the sum of the individual masses. We conclude that this difference is due to projection effects.

In addition, enough emission was detected in the northeast galaxy to observe specific characteristics therein. In this galaxy, the isophotes of the continuum show signs of a stellar bar; the velocity field shows no symmetry with respect to the minor axis, which again suggests that this galaxy has a stellar bar. It was also possible to conclude that the arms of the NGC $6090 \mathrm{NE}$ galaxy rotate in a trailing direction.

We concluded that the photometric centre of the southwest galaxy is in a location different from those reported of radio and infrared wavelengths, which were inconsistent. Furthermore, the maximum of ionized gas emitting at $\mathrm{H} \alpha$ and the maximum of the $2.22 \mu \mathrm{m}$ coincide, confirming that this knot is part of the galaxy, and therefore cannot be a field star. However, we were not able to study the morphological characteristics of the southwestern galaxy due to the size of the emission area that was detected. 
As regards optical wavelength images of NGC 6090, the system is similar to the Antennae (NGC 4038/39), while also it has been defined as a merger. In this paper we have discussed the kinematic differences between these galaxy systems from observations made at the same wavelength with similar instruments. This allowed us to conclude that their differences are mainly due to the stage of interaction of the galaxies that make up these systems. Through the kinematic analysis of NGC 6090 we concluded that these galaxies are in a stage of merger earlier than that of the galaxies in the Antennae.

Our research was carried out thanks to the Program UNAM-DGAPA-PAPIIT IN109919.

The authors express their gratitude to the Mexican National Council for Science and Technology (CONACYT) for financing this paper through project CY-109919.

This paper is based on observations performed with the scanning Fabry-Perot interferometer, PUMA, at the Observatorio Astronómico Nacional on Sierra San Pedro Mártir (OAN-SPM), Baja California, México.

We thank the day - and night time support staff at OAN-SPM for facilitating and helping us to obtain our observations.

The Digitized Sky Surveys were produced at the Space Telescope Science Institute under U.S. Government Grant NAG W-2166. The images of these surveys are based on photographic data obtained using the Oschin Schmidt Telescope on Palomar Mountain and the UK Schmidt Telescope. The plates were processed into the present compressed digital form with the permission of these institutions.

\section{REFERENCES}

Acosta-Pulido, J. A., Klaas, U., Laureijs, R. J., et al. 1996, A\&A, 315, L121

Amram, P., Marcelin, M., Boulesteix, J., \& Le Coarer, E. 1992, A\&A, 266, 106

Amram, P., Plana, H., Mendes de Oliveira, C., Balkowski, C., \& Boulesteix, J. 2003, A\&A, 402, 865

Arp, H. 1966, ApJS, 14, 1

Barnes, J. E. 1988, ApJ, 331, 699

Barnes, J. E. \& Hernquist, L. 1992, ARA\&A, 30, 705

Binney, J. \& Tremaine, S. 1987, Galactic dynamics, Princeton, N.J. : Princeton University Press

Bosma, A. 1981, AJ, 86, 1825

Bryant, P. M. \& Scoville, N. Z. 1999, AJ, 117, 2632

Cárdenas-Martínez, N. \& Fuentes-Carrera, I. 2018, ApJ, 868,141
Chamberlain, J. W. 1961, Physics of the aurora and airglow, International Geophysics Series, New York: Academic Press

Chisholm, J., Tremonti, C. A., Leitherer, C., Chen, Y., \& Wofford, A. 2016a, MNRAS, 457, 3133

Chisholm, J., Tremonti, C. A., Leitherer, C., Chen, Y., Wofford, A., \& Lundgren, B. 2015, ApJ, 811, 149

Chisholm, J., Tremonti Christy, A., Leitherer, C., \& Chen, Y. 2016b, MNRAS, 463, 541

Combes, F., Boisse, P., Mazure, A., Blanchard, A., \& Seymour, M. 2002, Galaxies and cosmology, 2nd ed. by F. Combes et al. (M. Seymour, Trans.). New York: Springer

Condon, J. J., Helou, G., Sanders, D. B., \& Soifer, B. T. 1990, ApJS, 73, 359

Cortijo-Ferrero, C., González Delgado, R. M., Pérez, E., et al. 2017, MNRAS, 467, 3898

Dinshaw, N., Evans, A. S., Epps, H., Scoville, N. Z., \& Rieke, M. 1999, ApJ, 525, 702

Epinat, B., Amram, P., Marcelin, M., et al. 2008, MNRAS, 388, 500

Fuentes-Carrera, I., Cárdenas-Martínez, N., SánchezCruces, M., 2015, IAUS, 309, 314

Fuentes-Carrera, I., Rosado, M., Amram, P., et al. 2004, A\&A, 415, 451

Fuentes-Carrera, I., Rosado, M., Amram, P., Salo, H., \& Laurikainen, E. 2007, A\&A, 466, 847

Gabbasov, R. F., Rosado, M., \& Klapp, J. 2014, ApJ, 787,39

Günthardt, G. I., Díaz, R. J., \& Agüero, M. P. 2016, AJ, 152,150

Hubble, E. 1936, ApJ, 84, 517

Hummel, E., van der Hulst, J. M., Keel, W. C., \& Kennicutt, Jr., R. C. 1987, A\&AS, 70, 517

Karachentsev, I. 1987, Double galaxies

Krassovsky, V. I., Shefov, N. N., \& Yarin, V. I. 1962, Atlas of the airglow spectrum 3000-12400 A

Lequeux, J. 1983, A\&A, 125, 394

Makarov, D., Prugniel, P., Terekhova, N., Courtois, H., \& Vauglin, I. 2014, A\&A, 570, A13

Martin, C. L. \& Soto, K. T. 2016, ApJ, 819, 49

Martin, J. M., Bottinelli, L., Gouguenheim, L., \& Dennefeld, M. 1991, A\&A, 245, 393

Mazzarella, J. M. \& Boroson, T. A. 1993, ApJS, 85, 27

Mihalas, D. \& Binney, J. 1981, Galactic astronomy: Structure and kinematics, 2nd edn.

Mihos, C. 2000, Galaxies: Interactions and Mergers, ed. P. Murdin, 2623

Miralles-Caballero, D., Colina, L., Arribas, S., \& Duc, P.-A. 2011, AJ, 142, 79

Mo, H., van den Bosch, F. C., \& White, S. 2010, Galaxy Formation and Evolution

NASA/IPAC. 2014, NASA/IPAC Extragalactic Database (NED), https://ned.ipac.caltech.edu/

Noguchi, M. 1987, MNRAS, 228, 635

Pasha, I. I. 1985, Soviet Astronomy Letters, 11, 1

Renaud, F., Boily, C. M., Fleck, J.-J., Naab, T., \& Theis, C. 2008, MNRAS, 391, L98 
Renaud, F., Bournaud, F., \& Duc, P.-A. 2015, MNRAS, 446, 2038

Repetto, P., Rosado, M., Gabbasov, R., \& FuentesCarrera, I. 2010, AJ, 139, 1600

Rosado, M., Gabbasov, R., \& Fuentes-Carrera, I. 2011, IAUS, 277, 259

Rosado, M., Gabbasov, R. F., Repetto, P., et al. 2013, AJ, 145, 135

Rosado, M., Langarica, R., Bernal, A., et al. 1995, $\mathrm{RMxAC}, 3,263$

Salo, H. \& Laurikainen, E. 2000a, MNRAS, 319, 377 2000b, MNRAS, 319, 393

Sanders, D. B., Scoville, N. Z., \& Soifer, B. T. 1991, ApJ, 370,158

Schweizer, F. 1986, Science, 231, 227

Sugai, H., Davies, R. I., Ishii, M., \& Ward, M. J. 2000,
MNRAS, 317, 447

Sugai, H., Hattori, T., Kawai, A., et al. 2004, ApJ, 615, L89

Teyssier, R., Chapon, D., \& Bournaud, F. 2010, ApJ, 720, L149

Toomre, A. \& Toomre, J. 1972, ApJ, 178, 623

Torres-Flores, S., Amram, P., Mendes de Oliveira, C., et al. 2014, MNRAS, 442, 2188

Vollmer, B., Marcelin, M., Amram, P., Balkowski, C., Cayatte, V., \& Garrido, O. 2000, A\&A, 364, 532

Vorontsov-Velyaminov, B. A., Noskova, R. I., \& Arkhipova, V. P. 2001, A\&AT, 20, 717

Wang, J., Zhang, Q., Wang, Z., Ho, P. T. P., Fazio, G. G., \& Wu, Y. 2004, ApJ, 616, L67

Minerva Muñoz Sardaneta and Mónica Sánchez-Cruces: Aix Marseille Univ, CNRS, CNES, Laboratoire d'Astrophysique de Marseille (LAM). 38 rue F. Joliot-Curie, 13388 Marseille cedex 13 France (minerva.munoz@lam.fr).

Margarita Rosado: Instituto de Astronomía,Universidad Nacional Autónoma de México (UNAM). Apdo. Postal 70-264, 04510, Ciudad de México, México (margarit@astro.unam.mx). 